\title{
APPENDIX: a List of Robotic Telescopes
}

\author{
Frederic V. Hessman
}

Universitäts-Sternwarte, Geismarlandstr. 11, 37083 Göttingen, Germany

\begin{abstract}
In order to help the reader keep somewhat abreast of the rapid development in the field of robotic telescopes, the current list on the web available at the Göttingen MONET website ${ }^{1}$ is included here as an appendix.
\end{abstract}

The selection of sites in the following tables is probably not exhaustive I apologize to any colleagues who feel left out. I have not included systems which are nearly robotic (i.e. which need a local operator to get the system started each night) but have included proposed (i.e. unfunded) projects (e.g. ROBONET and NOT) in the hopes that they will be funded and eventually of more immediate interest. The following abbreviations are used:

APT : (usually) Automatic Photoelectric Telescope

B\&C : Boller \& Chivens

C14 : Celestron 14-inch Schmidt-Cassegrain

GRB : Gamma-Ray Bursts

NEO : Near-Earth Objects

TNO : Trans-Neptunian Objects = Kuiper-Belt Objects

${ }^{1}$ http://www.astro.physik.uni-goettingen.de/ hessman/MONET 

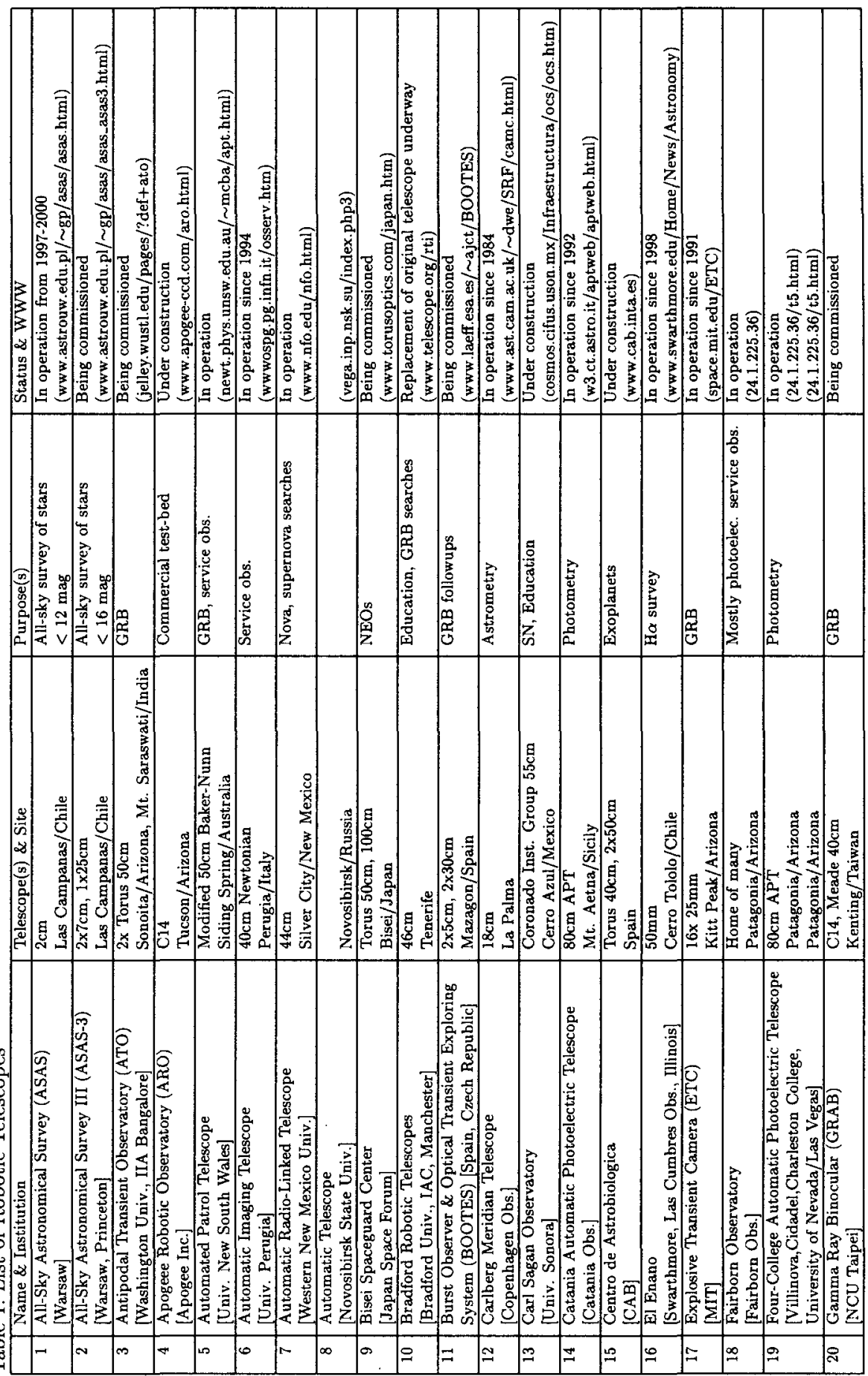


\begin{tabular}{|c|c|c|c|c|c|c|c|c|c|c|c|c|c|c|c|c|c|c|c|c|}
\hline & 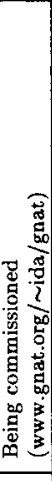 & 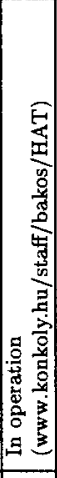 & 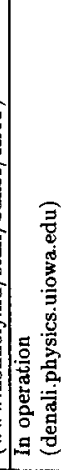 & \multicolumn{2}{|c|}{ 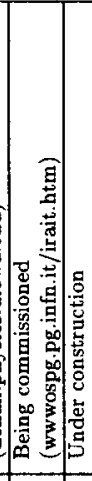 } & 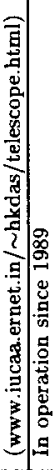 & 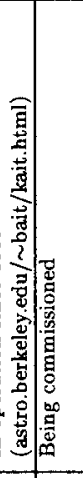 & 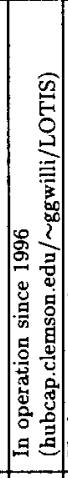 & \multicolumn{2}{|r|}{ 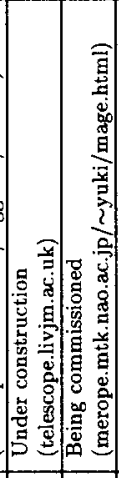 } & 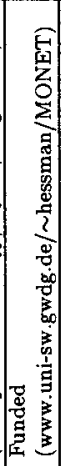 & 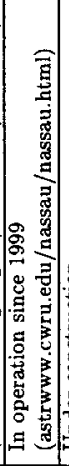 & \multicolumn{2}{|c|}{ 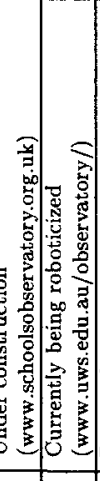 } & \multicolumn{2}{|c|}{ 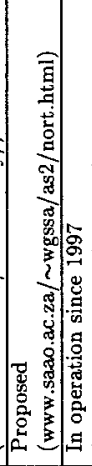 } & \multicolumn{2}{|c|}{ 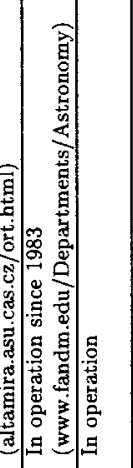 } & 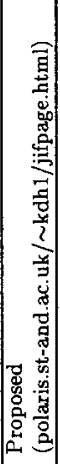 & 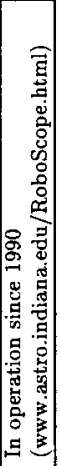 \\
\hline & & 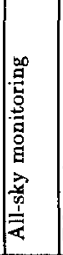 & 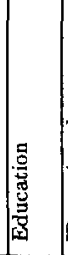 & 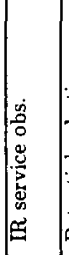 & 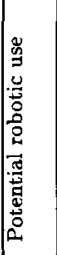 & 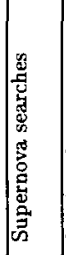 & 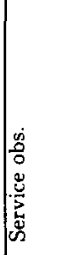 & 䊁 & $\mid$ & 焉 & 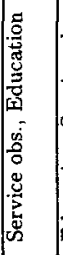 & 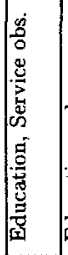 & 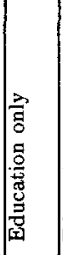 & 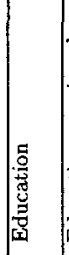 & 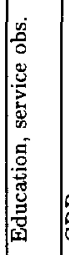 & 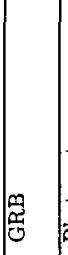 & 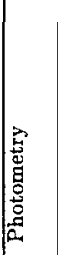 & 禹 & 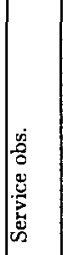 & 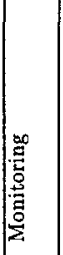 \\
\hline & 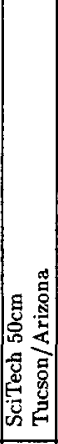 & 㕝 & 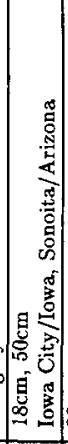 & 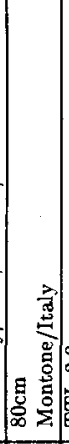 & 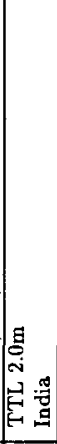 & 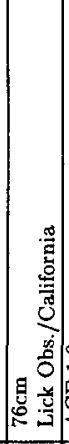 & 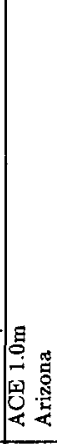 & 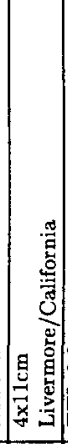 & 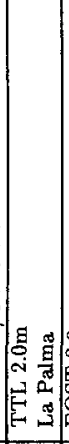 & 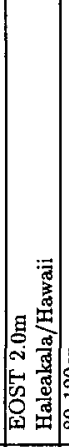 & 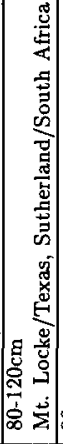 & 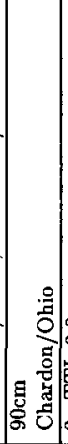 & 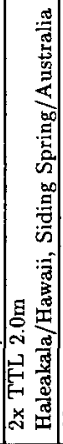 & . & 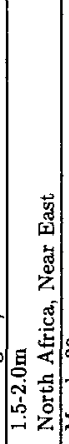 & 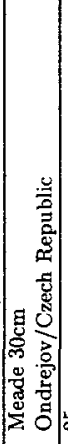 & 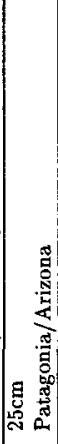 & 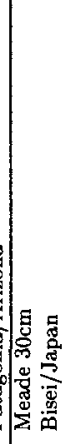 & 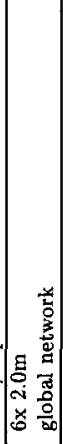 & 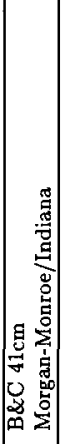 \\
\hline & 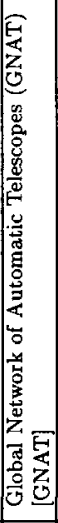 & 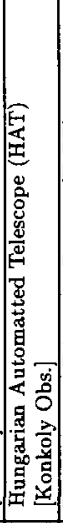 & 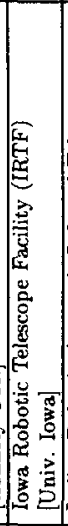 & 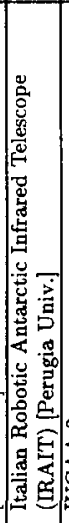 & 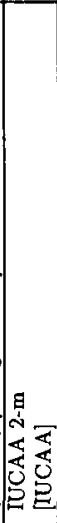 & 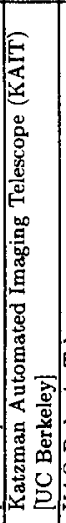 & 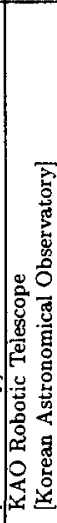 & 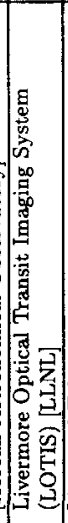 & 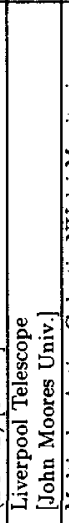 & 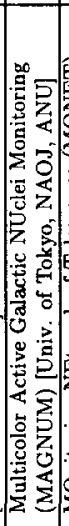 & 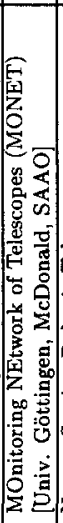 & 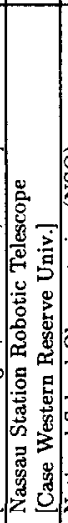 & 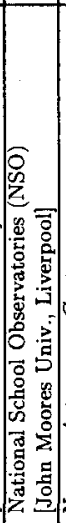 & 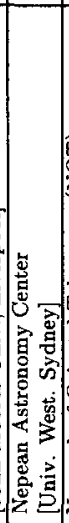 & 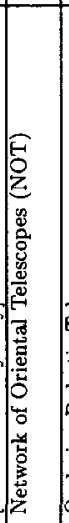 & 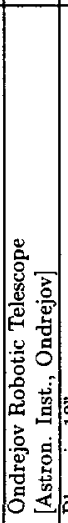 & 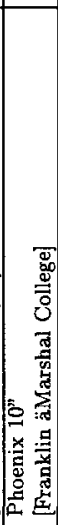 & 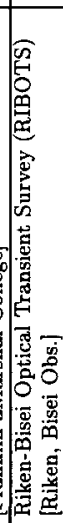 & 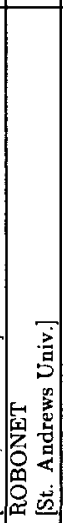 & 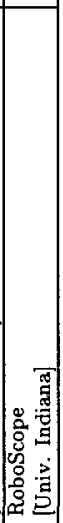 \\
\hline & ন & & $\mathscr{2}$ & & $\stackrel{2}{2}$ & & & & & ల్ల & & & & & & & & & & \\
\hline
\end{tabular}



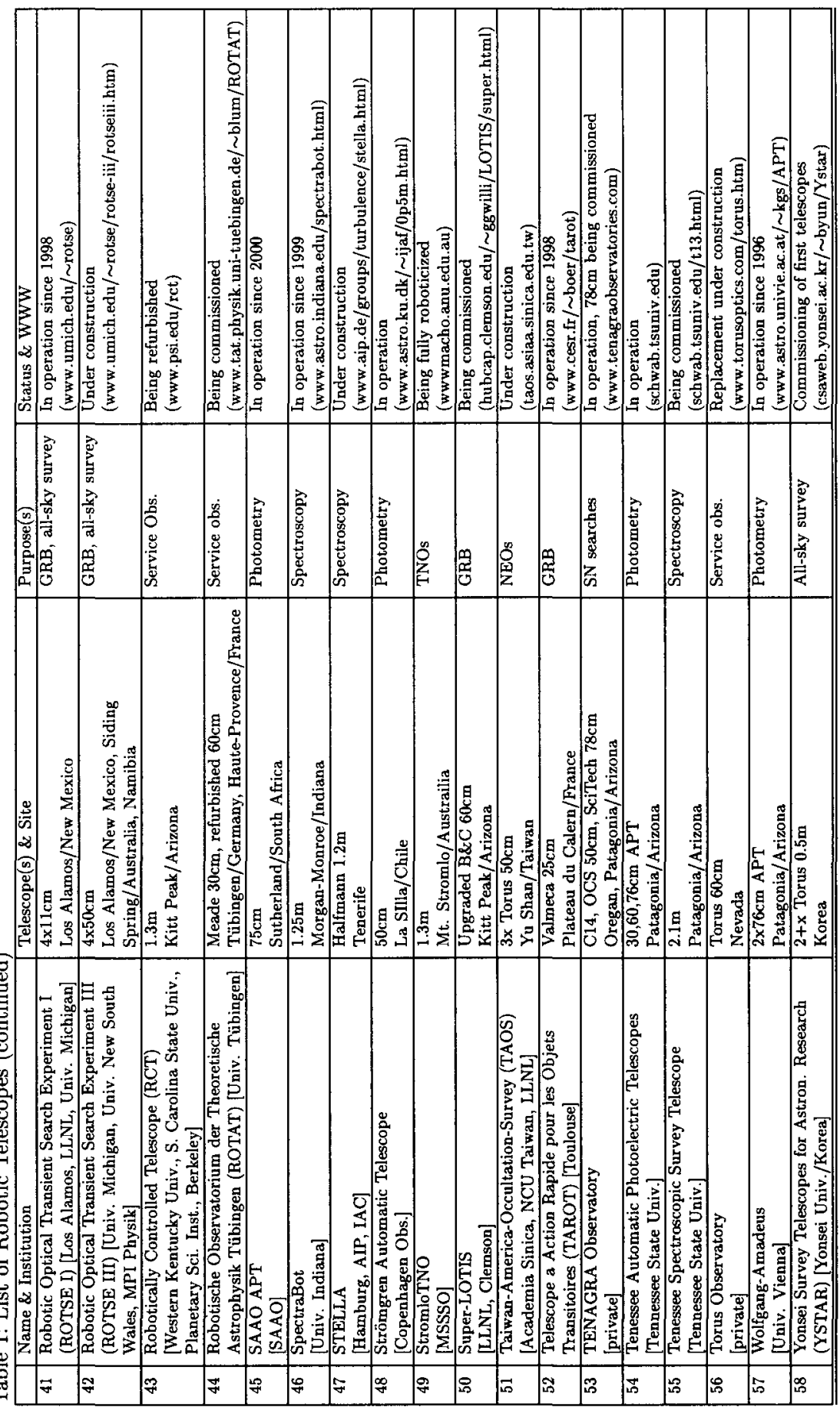